\title{
STUDY COMPARING MATERNAL SERUM URIC ACID AND CALCIUM VERSUS UMBILICALARTERY DOPPLER IN PREECLAMPSIA
}

Tarek Abd Elzaher Karkor, Doaa Ali Abdelmonsif*, Tamer Mohamed Abdel-Aziz, Rawan Gamal Elsayed Asala Department of Obstetrics and Gynecology, Department of Medical Biochemistry*, Faculty of Medicine, University of Alexandria

\section{Introduction}

Preeclampsia is a pregnancy-specific disorder and one of the major causes of maternal and perinatal morbidity and mortality worldwide.

There have been many screening tests evaluated in the literature over the years for There have been mans

Umbilical artery Doppler assessment has been shown to reduce perinatal mortality and morbidity in high-risk obstetric situations.

The umbilical arterial waveform usually has a "saw tooth" pattern with flow always in the forward direction, which is towards the placenta. An abnormal waveform shows absent or reversed diastolic flow.

Evidence supporting an inverse relationship between calcium intake and blood pressure has strengthened in the past decade. The role of calcium in gestational hypertension is still not clear.

Increased uric acid level is a key clinical feature of preeclampsia; higher levels correlate with significant maternal and fetal morbidity and mortality.

\section{Aim of the work}

The aim of this work was to study the role of maternal serum uric acid and calcium versus Umbilical artery Doppler blood flow in preeclamptic patients at 28-30 weeks of gestation compared to Normotensive pregnant subjects at El-Shatby Maternity University Hospital.

\section{Patients and Methods}

This study was carried out on 90 cases admitted to El-Shatby Maternity University Hospital

The study was conducted on two groups of pregnant women:

Group I: Control: 45 normal healthy pregnant women at $28-30$ weeks of gestation.

Group I: Control: 45 normal healthy pregnant women at $28-30$ weeks of gestation.
Group II: Cases: Including 45 pregnant women who and they further divided into

Group II: Cases: Including 45 pregnant women who and they fur
Mild Preeclampsia $(\mathrm{n}=20)$ Severe Preeclampsia $(\mathrm{n}=25)$

Umbilical artery Doppler (S/D ratio, RI, PI) was assessed for both groups
Laboratory investigations including:

- Serum total calcium concentration was determined by colorimetric test using Arsenazo - III (Sigma Aldrich, USA).

- Serum uric acid level measurement by enzymatic color test using Uricase and Peroxidase enzymes. The analyzer used was "SIEMENS Dimension clinical chemistry system".

\section{Results}

Table 1: sensitivity, specificity and accuracy of serum calcium and uric acid to predict severe preeclampsia from mild preeclampsia.

\begin{tabular}{|c|c|c|c|c|c|c|c|c|}
\hline & AUC & $\mathbf{P}$ & 95\% C.I & $\begin{array}{l}\bar{t} \\
\bar{z}\end{array}$ & 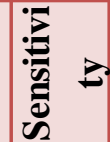 & $=$ & 主 & $\frac{2}{z}$ \\
\hline um calcium (mg/dl) & 0.92 & $0.001^{*}$ & $0.66-0.93$ & $\leq 7.2$ & 95.0 & 90.0 & 92.0 & 93.0 \\
\hline Serum uric acid (mg/dl) & 0.80 & $0.008^{*}$ & $0.320-0.9$ & $>5.6$ & 82.0 & 70.0 & 82.6 & 81.0 \\
\hline
\end{tabular}

AUC: Area Under a Curve $p$ value: Probability value CI: Confidence Intervals NPV: Negative predictive value PPV: Positive predictive value

*: Statistically significant at $\mathrm{p} \leq 0.05$ Table 2: sensitivity, specificity and accuracy of Doppler to predict severe preeclampsia from mild
preeclampsia.

\begin{tabular}{|l|c|c|c|c|c|c|c|c|}
\hline Doppler & AUC & p & 95\% C.I & Cut off & Sensitivity & Specificity & PPV & NPV \\
\hline
\end{tabular}

\begin{tabular}{|l|c|c|c|c|c|c|c|c|}
\hline UAPI & 0.90 & $<0.001^{*}$ & $0.929-1.0$ & $>1.13$ & 83.0 & 88.0 & 80.6 & 81.0 \\
\hline
\end{tabular}

Table 3: Validity (AUC, sensitivity, specificity) for Serum calcium + Serum uric acid + umbilical artery Doppler to predict severe preeclampsia $(n=25)$ from mild preeclampsia $(n=20)$

\begin{tabular}{|l|c|c|c|c|c|c|c|}
\hline & AUC & p & $\mathbf{9 5 \%}$ C.I & Sensitivity & Specificity & PPV & NPV \\
\hline $\begin{array}{l}\text { Serum calcium + Serum uric acid } \\
\text { + Doppler }\end{array}$ & 0.98 & $<0.001^{*}$ & $1.0-1.0$ & 98.0 & 100.0 & 97.0 & 99.0 \\
\hline
\end{tabular}

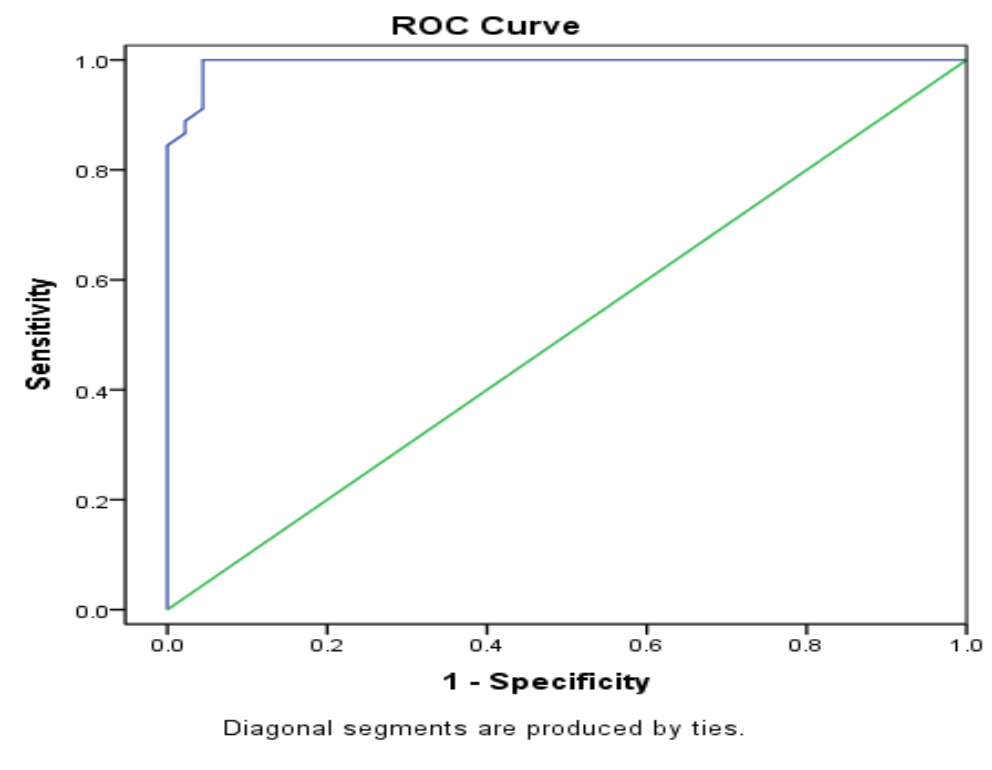

Figure: ROC curve for Serum calcium + Serum uric acid + umbilical artery Doppler to predict severe preeclampsia $(\mathrm{n}=25)$ from mild preeclampsia $(\mathrm{n}=20)$

\section{Conclusion}

- There was significant decrease in serum calcium level, while there was significant increase in serum uric acid level and umbilica artery Doppler parameters among preeclampsia group.

- In conclusion, it is more convincing to take on that no single biomarker will attain $100 \%$ sensitivity and specificity and that combination of different biomarkers together and umbilical artery Doppler indices with clinical data is more expected to be used in clinical practice.

2021 OAlexandria Faculty of Medicine
CC-BY-NC

